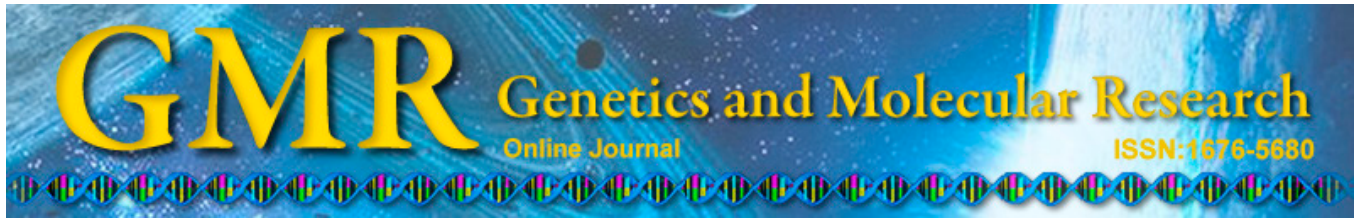

\title{
Treatment of vertebral body compression fractures using percutaneous kyphoplasty guided by a combination of computed tomography and C-arm fluoroscopy with finger-touch guidance to determine the needle entry point
}

G.Y. Wang ${ }^{1}$, C.C. Zhang' ${ }^{2}$ K. Ren ${ }^{3}$, P.P. Zhang ${ }^{1}$, C.H. Liu' ${ }^{1}$, Z.A. Zheng', Y. Chen ${ }^{1}$ and R. Fang ${ }^{1}$

${ }^{1}$ Department of Orthopedics, Wuhu No. 2 People's Hospital, Wannan Medical College, Anhui, China

${ }^{2}$ Department of Orthopedics, Changhai Hospital, Second Military Medical University of Chinese PLA, Shanghai, China ${ }^{3}$ Department of Orthopedics,

Gen Hospital of Nanjin Military Area Command, Nanjin, Jiangsu, China

Corresponding author: G.Y. Wang

E-mail: wangguangye@126.com

Genet. Mol. Res. 14 (1): 1546-1556 (2015)

Received December 19, 2013

Accepted May 28, 2014

Published March 6, 2015

DOI http://dx.doi.org/10.4238/2015.March.6.2

\begin{abstract}
This study aimed to evaluate the results and complications of image-guided percutaneous kyphoplasty (PKP) using computed tomography (CT) and C-arm fluoroscopy, with finger-touch guidance to determine the needle entry point. Of the 86 patients (106 PKP) examined, 56 were treated for osteoporotic vertebral compression fractures and 30 for vertebral tumors. All patients underwent imageguided treatment using CT and conventional fluoroscopy, with finger-
\end{abstract}


touch identification of a puncture point within a small incision (1.5 to $2 \mathrm{~cm}$ ). Partial or complete pain relief was achieved in $98 \%$ of patients within $24 \mathrm{~h}$ of treatment. Moreover, a significant improvement in functional mobility and reduction in analgesic use was observed. CT allowed the detection of cement leakage in $20.7 \%$ of the interventions. No bone cement leakages with neurologic symptoms were noted. All work channels were made only once, and bone cement was distributed near the center of the vertebral body. Our study confirms the efficacy of PKP treatment in osteoporotic and oncological patients. The combination of $\mathrm{CT}$ and $\mathrm{C}$-arm fluoroscopy with finger-touch guidance reduces the risk of complications compared with conventional fluoroscopy alone, facilitates the detection of minor cement leakage, improves the operative procedure, and results in a favorable bone cement distribution.

Key words: Percutaneous kyphoplasty; Spine fractures; Osteoporosis; Computed tomography; C-arm fluoroscopy; Spine neoplasms

\section{INTRODUCTION}

Percutaneous kyphoplasty (PKP) is a minimally invasive, imaging-guided technique used to inject bone cement into a vertebral body to reinforce fracture sites and relieve pain. PKP is initially used to treat primary spinal tumors and subsequently used to treat metastatic spinal tumors, spinal osteoporotic compression fractures, myeloma, and other rare disorders causing bone pain, such as histocytosis and osteogenesis imperfecta (Hide and Gangi, 2009). As the operation materials and image-guided techniques are constantly improving, PKP has been greatly modified in terms of patient selection and operation procedures (Kallmes and Jensen, 2003).

Currently, vertebroplasty is mostly conducted under the guidance of a C-arm X-ray machine or computed tomography (CT); however, the puncture process mostly depends on the experience and skill of the operator. Therefore, vertebroplasty has certain disadvantages. For the safety of vertebroplasty centesis, some studies have been conducted locally and abroad. In addition, local and international research has focused on vertebroplasty guided by $\mathrm{C}$-arm fluoroscopy, CT, and a combination of C-arm fluoroscopy and CT. As the puncture process is operator-dependent, several shortcomings limit the clinical application of the current technique, such as non-uniform bone cement distribution, inaccurate location, bone cement leakage, puncture failure, vertebral body refracture, and numerous complications caused by X-ray radiation affecting patients and operators (Anselmetti et al., 2011; Smith et al., 2012; Trumm et al., 2012a). The above complications are usually closely related to the operation technique. The search for a modified centesis that can accurately complete a specific puncture is a research hotspot in vertebroplasty (Shengzhong et al., 2012). Domestic and foreign scholars have used multiple imaging equipment such as vertebroplasty guided by bilateral puncture C-arm fluoroscopy, CT, a combination of three-dimensional (3-D) CT and C-arm fluoroscopy, and real-time 3-D navigation to guide vertebroplasty (Katsanos et al., 2010; Rad et al., 2011; Rad and Kallmes, 2011; Trumm et al., 2012a).

Although various clinical effects were observed in the above studies, several short- 
comings were noted. First, although percutaneous puncture guided by CT is accurate, the operation is very complex and lengthy. In addition, dynamic monitoring is impossible, and the promotion and application of the method in clinics is difficult. Second, the efficiency of the C-arm mostly depends on the operator's experience and skill. Third, the navigation system easily causes image drift, which is an error involving deviation from the real position caused by a drift of intraoperative tissue structures. Therefore, surgeons who use navigation systems during vertebroplasty must understand their basic working principles, characteristics, and drawbacks to minimize discrepancies in the navigation information. Concurrently, surgeons must have good experience with this navigation system. When necessary, the traditional technique is used. Therefore, the value of the system is appreciated. Finally, the working channel is not constructed at one time. The puncture point is usually at the bone crest (lumbar herring bone crest) or bone gap (junction of the thoracic vertebrae rib and transverse process); thus, guidance using extremely accurate images to avoid needle-tip drift during puncturing is also difficult. Repeated puncture at the needle entry site and repeated confirmation using imaging increases operation time. Moreover, repeated puncture can easily cause bone cement leakage and direct injuries. Therefore, the creation of the working channel is not suitable for wide clinical application and development (Chew et al., 2011). As current image-guided methods are difficult to use in clinical practice, new methods need to be developed.

The use of C-arm fluoroscopy in vertebroplasty allows monitoring of the head-tilt angle during percutaneous puncture in the lateral position, and dynamic monitoring of bonecement filling. CT-guided puncture is very accurate for observing foci and vertebral body structures, and needle entry can be planned based on the CT images. The needle entry point is usually at the bone crest or bone gap, superior articular process junction of the lumbar vertebra, and transverse process or transverse process rib clearance of the thoracic vertebra. The bony anatomical landmarks are more obvious. Although the needle entry point in the operative field cannot be completely exposed in minimally invasive interventions, it can be determined by touching the bony anatomical landmarks.

Based on the above factors, the purpose of this study was to evaluate the clinical results and complications of a novel approach to image-guided PKP involving a combination of $\mathrm{CT}$ and $\mathrm{C}$-arm fluoroscopy with the finger-touch method to identify the needle entry point.

\section{MATERIAL AND METHODS}

\section{Objects}

This study was conducted in accordance with the principles of the Declaration of Helsinki after approval from the Ethics Committee of Wuhu No. 2 People's Hospital. Written informed consent was obtained from all participants. Eighty-six patients treated in our hospital (106 vertebral bodies) from October 2007 to February 2011 were retrospectively studied. Among these patients, 56 (65 vertebral bodies) presented osteoporotic vertebral body compression fractures (14 thoracic vertebrae and 41 lumbar vertebrae; aged 55 to 87 years) and 30 (41 vertebral bodies) presented vertebral tumors (20 thoracic vertebrae and 21 lumbar vertebrae; aged 41 to 78 years) (Table 1). Patient selection was jointly performed by internists, oncologists, osteopathologists, and radiologists specialized in metabolic bone diseases. In addition, the selection criteria were based on those by the American College of Radiology (20002001). Indications for PKP were as follows: osteoporosis-induced spinal fracture of the lum- 
bar back, intractable chest/back pain, and spinal osteolytic destruction, suggesting that internal medicine treatment was ineffective (rest, anodyne, waistline fixation); and a spinal tumor.

Table 1. Basic demographic features of the patients.
\begin{tabular}{llccrcccc}
\hline Group & N & Age (years) & Age range (years) & Male & Female & Cone number & Thoracic vertebra & Lumbar vertebra \\
\hline Osteoporosis & 56 & $67.1 \pm 8.3$ & $55-87$ & 9 & 47 & 65 & 24 & 41 \\
Cancer & 12 & $63.5 \pm 8.1$ & $41-78$ & 11 & 0 & 17 & 12 & 5 \\
Total & 68 & & & 20 & 47 & 82 & 36 & 46 \\
\hline
\end{tabular}

Age is reported as means $\pm \mathrm{SD}$.

\section{Methods}

Informed consent was obtained from all patients after explaining the benefits and risks of the technique. To minimize infection risk, prophylactic antibiotics were used in patients who received cancer treatment 3 days prior to surgery.

All patients underwent PKP guided by a combination of $\mathrm{CT}$ and $\mathrm{C}$-arm with the fingertouch method to identify the needle entry point; the operation was completed in an X-ray/CT room. In addition, all patients received local anesthesia and unilateral punctures. Before the operation, grids $(0.5 \times 0.5 \mathrm{~cm})$ were closely applied on the skin above the vertebral bodies, and simple scanning using CT was performed to select the optimal puncture level. Under infrared radiation, the vertical projection points of the skin puncture points were marked by grids. The operative field and towel were conventionally disinfected. Based on the skin projection points, a small, lateral transverse incision was made. An incision measuring 1.5 to $2.0 \mathrm{~cm}$ long was gently made using a sharp blade to cut open the fasciae transversely. Subsequently, a straight vascular clamp was used for vertical insertion into the back muscles along the puncture points to identify the needle entry points. In obese patients, identifying the needle entry points using the operator's fingers was relatively difficult. Therefore, the skin incision was slightly expanded to allow the needle entry point to be easily felt using the fingers after blunt separation of the muscle gap; the forefinger of the operator contacted the needle entry point. For the lumbar vertebrae, the corner of the small processus articularis is the preferred entry point; the transverse process is used. At the lumbar puncture point, the finger could reach the upper articular process on the highest punctured vertebra, the outward transverse process in the partial depth, the transverse process ridge at the lower outer portion in depth, and the lamina ridge and articular process in the inner lower portion. The transverse ridge and lamina constituted a herringbone crest, which is the puncture point. Guided by the operator's finger, the needle was inserted at the point by touching the herringbone crest. The needle entry point could also be selected according to the angle on the intraoperative CT image, with slight adjustment to the inside/outside and upper/lower margins. In addition, in terms of the thoracic vertebrae, the puncture point was selected to occupy one-third of the gap at the junction of the transverse process. The rib pedicle of the vertebral arch using a lateral approach was preferentially used. Furthermore, the hand was at the junction of the transverse process and the upper one-third of the rib. Under finger guidance, the puncture needle reached the puncture point and entered the cortex. Subsequently, the guide needle was led into the outer cortex. The abduction angle and position were determined using a single CT scan. The unsatisfactory abduction angles could be slightly adjusted using a fine puncture needle with an oblique mouth until satisfactory angles were obtained. The position of the CT bed (height and depth) was recorded during 
CT. The CT bed was repositioned until the vertebra was located under the control position of the lateral C-arm; the position of the CT bed (height and depth) was recorded when the C-arm was being monitored. The $\mathrm{C}$-arm $\mathrm{X}$-ray machine was used to determine the head tilt angle and height. When the abduction angle was satisfactory, percutaneous puncture was performed directly using the guide needle. The $\mathrm{CT}$ bed was moved to the $\mathrm{CT}$ position. CT was conducted to determine the angle and location. When the abduction angle was unsatisfactory, a fine obliquemouth puncture needle was used to make a fine adjustment. When the abduction angle was satisfactory, the puncture was performed directly using the guide needle. The X-ray machine and single-layer CT scanner were again used to confirm that the guide needle reached the ideal entry point, needle head-tilt angle, and abduction angle. The CT bed was electrically adjusted to the control position under the $\mathrm{C}$-arm through the guide needle. With the guide needle at the center, peripheral sclerotin was gradually dilated and compressed to closely circumvent the puncture tube until the puncture needle was pressed by the working cannula to the anterior one-third middle line of the affected vertebra. Under intermittent perspectivity, the vertebral body was dilated and/or bone cement was filled. Subsequently, CT was performed to examine the bone-cement distribution pattern. During CT, the doctors left the CT room to minimize exposure to radiation. All operations were performed by a single operator to minimize differences between operators with the use of the finger-touch method.

\section{Curative effect evaluation}

For all patients, data were collected according to the following series of criteria. Questionnaires were prepared for preoperative and postoperative 24-h evaluations. For the evaluation of preoperative pain, a 10-point visual analogue scale (VAS; most algetic: 10 points; painless: 1 point) was used. Four-point functional activity evaluation (bed rest: 4 points; wheelchair use: 3 points; limited pain during walking: 2 points; normal walk: 1 point) and 3-point anodyne use scoring (anodyne used for the whole day: 3 points; anodyne used in the daytime: 2 points; occasional use: 1 point) were also used.

\section{Statistical analysis}

The SPSS 15.0 statistics software was used for data analysis, and measurement data were reported as means $\pm \mathrm{SD}$. An independent sample $t$-test was used for the comparison of 2 groups of independent samples. The paired $t$-test was used for before and after treatment comparison between the 2 groups. The chi-square test was used for the comparison of count data between the 2 groups. If $\mathrm{P}<0.05$, no statistically significant difference existed.

\section{RESULTS}

Clinical symptoms and imaging findings were used to confirm indication for PKP. One hundred and six cases of vertebral body compression fractures were noted (imaging manifestations of typical cases are shown in Figure 1). Every patient underwent PKP of 1 to 3 vertebral bodies, with a mean 1.2 vertebral bodies per patient. A unilateral puncture was performed for all vertebral bodies, which was completed in the first attempt in the majority of vertebral bodies $(90 \%)$. In addition, the working channel was dilated grade-by-grade and constructed once. The mean operation time per vertebral body was $25 \pm 5 \mathrm{~min}$, and bone cement was filled 


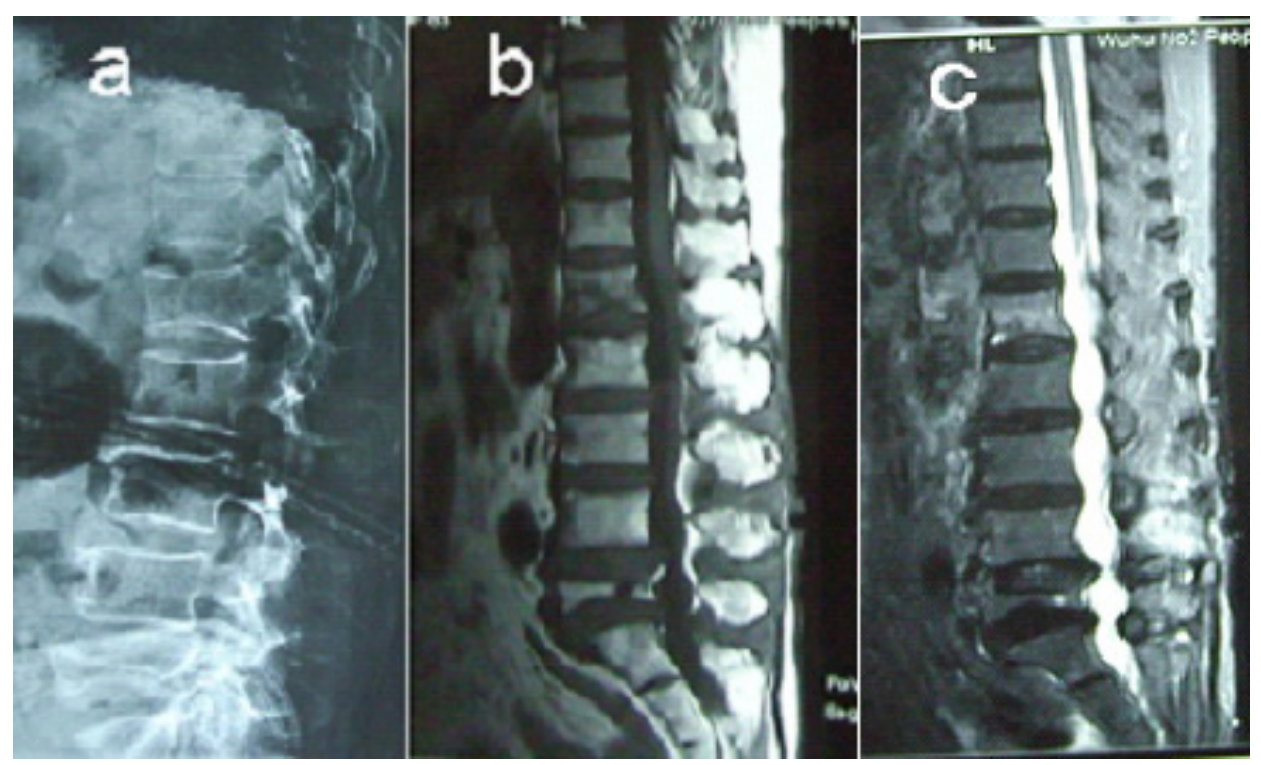

Figure 1. An 83-year-old woman with osteoporosis. Anatomical compatibility for percutaneous kyphoplasty (PKP) of L1 and incompatibility for PKP of L5. (a) In the lateral radiographic view, the height of the compression fracture of the L5 vertebral body is more than one-third of the original one. The heights of the other lumbar vertebral bodies are normal. (b, c) The sagittal magnetic resonance images obtained with turbo spin-echo T1-weighted (b) and fatsuppressed short tau inversion recovery (STIR) T2-weighted (c) sequences show a change in signal intensity at the L1 vertebral body, characterized by T1 hypointensity and STIR T2 hyperintensity owing to bone marrow edema resulting from a recent vertebral collapse that was causing pain (clinical correspondence). The signal intensity at L5 (old vertebral compression fracture) is normal.

in the center of $95 \%$ of vertebral bodies (Figure 2). Postoperatively, braces were worn for protection.

According to the clinical results, pain was apparently relieved or completely relieved in all patients (98\%) within $24 \mathrm{~h}$. Among the 86 patients, 56 presented osteoporosis and 30 vertebral tumors. Pre- and postoperative pain, functional activities, and anodyne use were evaluated (Table 2). The mean VAS, activity, and anodyne use scores decreased postoperatively compared with the preoperative scores $(2.81 \pm 1.33$ vs $8.55 \pm 0.72,1.12 \pm 0.59$ vs 2.97 \pm 0.63 , and $1.17 \pm 0.31$ vs $2.95 \pm 0.20$, respectively). At $24 \mathrm{~h}$ after PKP, significant differences were noted in pain recovery and anodyne use $(\mathrm{P}<0.01)$.

Two cases of mild complications were noted in our study, which were hematoma caused by the puncture and subcutaneous swelling of the puncture incision. In addition, postoperative radiotherapy and percutaneous drainage were performed. The complications resolved with application of pressure dressings.

Of the 86 patients, $22(20.7 \%)$ experienced bone cement leakage outside the spinal vertebral body, including 12 patients with vertebral tumors and 10 patients with osteoporotic collapsing fractures. The leakage occurred in the paravertebral veins (3 cases of vertebral tumors and one case of osteoporosis), outside the dura mater (7 cases of vertebral tumors and 5 cases of osteoporosis), in the paravertebral soft tissues (4 cases of vertebral tumors and one case of osteoporosis), and in the nerve root exit zone (one case of vertebral tumor); and no patient experienced bone cement leakage caused by the puncture. 


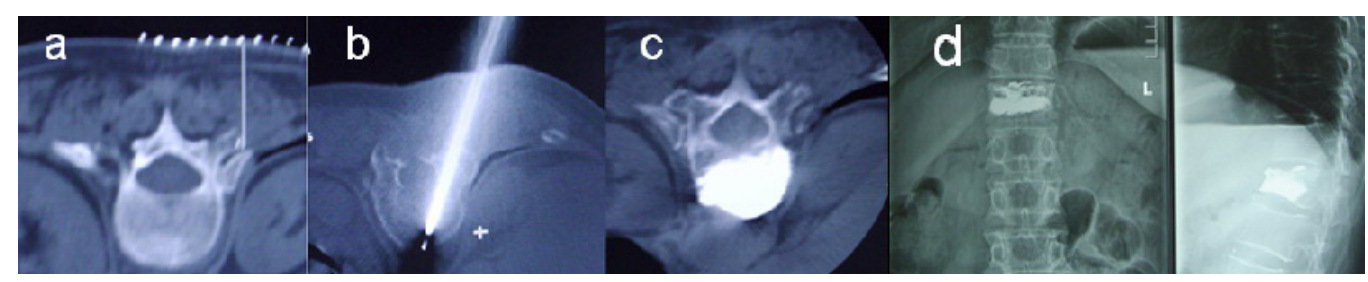

Figure 2. Dorsal percutaneous kyphoplasty with the unilateral transcostovertebral approach. A 65-year-old woman with osteoporosis. (a) Computed tomography (CT) targeting of D12 with tagging using a metal mesh on the skin. (b) C-arm fluoroscopy-guided puncture needle in the center of the vertebral body. (c) Post-procedural CT scan demonstrates even bone cement distribution without leakage. (d) Post-procedural radiograph demonstrates satisfactory bone cement distribution without leakage.

Table 2. Comparison of patient score results between groups (means \pm SD).

\begin{tabular}{|c|c|c|c|c|c|c|c|c|c|c|}
\hline & \multicolumn{3}{|c|}{ Total $(\mathrm{N}=86)$} & \multirow[t]{2}{*}{ P value* } & \multicolumn{2}{|c|}{ Osteoporosis $(\mathrm{N}=56)$} & \multirow[t]{2}{*}{ P value* } & \multicolumn{2}{|c|}{ Cancer $(\mathrm{N}=30)$} & \multirow[t]{2}{*}{ P value* } \\
\hline & Scale & Pre-PKP & Post-PKP & & Pre-PKP & Post-PKP & & Pre-PKP & Post-PKP & \\
\hline & $1-10$ & $8.55 \pm$ & $2.81 \pm 1.3$ & & $8.51 \pm 0.64$ & $2.76 \pm 1$ & & $8.63 \pm 0.95$ & 2.85 & $<0.01$ \\
\hline & $1-4$ & $2.97 \pm 0$. & $1.12 \pm$ & & $2.91 \pm$ & $1.05 \pm$ & $<0$. & $3.18 \pm$ & $1.19=$ & $<0.01$ \\
\hline Analgesic & $1-3$ & $2.95 \pm 0.20$ & $1.17 \pm 0.31$ & $<0.01$ & $2.93 \pm 0.25$ & $1.02 \pm 0.37$ & $<0.01$ & $2.98 \pm 0.19$ & $1.21 \pm 0.47$ & $<0.01$ \\
\hline
\end{tabular}

*Represent the comparison between before and after treatment, $\mathrm{P}<0.01$ using the paired $t$-test. VAS: visual analogue scale; PKP: percutaneous kyphoplasty.

\section{DISCUSSION}

Our study investigated pain relief and vertebral body strengthening. The majority of patients presented osteoporosis compression fractures (61.3\%), and the remaining bone tumors $(38.7 \%)$. Recent studies have shown that PKP can relieve pain and effectively restore vertebral body height.

In terms of patient selection, consistency of the clinical evaluation (pain sites) with the imaging examination (structure change) is the most important indicator. The bone marrow edema signal in patients is complicated, with the latest spinal accumulated evidence indicated by the pain sites (Zampini et al., 2010), thereby regarded as the indication for conducting PKP (Figure 1). At the same time, questionnaire surveys are conducted before and after PKP.

The junction of the superior articulate process of the lumbar vertebra and transverse process rib clearance of the thoracic vertebra, which acts as the puncture needle entry point, and bony anatomical landmarks are more visible. In minimally invasive interventions, the operative field of the needle entry point cannot be completely exposed. However, determining the needle entry point by touching these bony anatomical landmarks is feasible.

The finger guidance method was used to determine the needle entry point by touching the anatomical landmarks of the needle entry point. Image drift will occur in operations guided by the traditional imaging system (CT and C-arm), which causes a shift in the puncture projection point. In addition, the puncture point is usually in the bone crest, bone gap, or bone transitional site, which often causes puncture needle tip slippage. Therefore, accurately finding the needle entry point is impossible, in case of blindsight. For quick determination of the correct puncture point and rapid insertion of the needle, a small incision and the use of the finger to touch the puncture point before puncture can be used, under the guidance of projection points on the skin. 
PKP was conducted for all cases under the guidance of a combination of CT and C-arm fluoroscopy, with the finger-touch method to identify the needle entry point. PKP is more accurate and safe than simple $\mathrm{CT}$ or $\mathrm{C}$-arm application. In addition, PKP is less time-consuming than combined CT and C-arm fluoroscopy. The CT and C-arm combination is very safe and effective in percutaneous centesis. Especially for those vertebral bodies with anatomic variations, combined CT and C-arm fluoroscopy is the optimal puncture approach. Although the optimal puncture angle and puncture approach can be accurately designed on the computer, in clinical practice, the puncture point is not as smooth as the needle entry point in the bone crest (lumbar herring bone crest) or bone gap (junction of the thoracic vertebrae rib and transverse process). Conducting a simple blind puncture is very difficult. Therefore, repeated punctures and multiple confirmations of the imaging device are necessary, which prolongs operation time and increases the risk of complications (Grossterlinden et al., 2009; Young et al., 2011). In the current study, the vertical projection point of the optimal needle entry point on the skin was marked using grids and infrared radiation; a small, transverse lateral incision 1.5 to $2.0 \mathrm{~cm}$ in length was made along the needle entry point; and the open fascias were transversely cut. In addition, a straight vascular clamp was subsequently used to conduct a blunt separation of the lumbar back muscles and touch the needle entry point. Guide puncture can be completed at the first attempt, and ideally involves an oblique incision and angular adjustment. Therefore, it can reduce puncture time, operation risk, and various complications caused by the puncture.

Because the vertical projection point of the optimal needle entry point on the skin is marked by grids and infrared radiation, only a finger can be inserted for the skin incision. For a simple blind puncture, the increased size of the skin incision is not apparent; however, a transverse incision of the skin and deep fascia can reduce the soft tissue barrier in case of a decumbent puncture. In addition, a unilateral puncture approach was used in all cases, which causes lesser damage than the bilateral puncture approach. For the lumbar vertebrae, an approach using the pedicle of the vertebral arch is preferentially used; however, a lateral approach using the pedicle of vertebral arch is used for thoracic vertebrae.

Improvements in puncture accuracy further change the treatment mode. In addition, unilateral and large angle puncture can prevent the shortcomings associated with central bone interval formation of the vertebral body, which is caused by the bilateral puncture, and reduce complications and trauma.

Previous studies on bone cement filling in vertebroplasty have been conducted at home and abroad. These studies investigated puncture accuracy and safety, and focused on safe bone cement filling (Hide and Gangi, 2009; Anselmetti et al., 2011; Smith et al., 2012; Trumm et al., 2012b). However, they do not prioritize higher requirements for the bone cement-filling sites. Therefore, clinical applications of these methods are limited. The method used for vertebral body compression fracture is associated with cuneate changes, and involves compression at one-third of the anterior and central vertebral bodies. Therefore, bone cement filling was performed at one-third anterior/central of the vertebral body, which can provide mechanical characteristics complying with the associated pathology and physiology. In addition, bone cement filling at the center can reduce bone cement leakage, improve safety of the bone cement filling, and provide better mechanical support because of increased distance and tissue intervals away from important peripheral tissues (Shengzhong et al., 2012). Compared with the lateral bone cement filling of a bilaterally punctured vertebral body, bone cement filling at the center has greater research value and application prospect. Therefore, PKP guided by a combination of CT and C-arm fluoroscopy, with the finger-touch method to determine the 
needle entry point, can ensure accurate puncture.

Bone cement filling is one the most important processes and requires real-time monitoring with C-arm fluoroscopy or CT to monitor bone cement distribution and bone cement leakage. Therefore, compared with simple C-arm fluoroscopy, CT can accelerate evaluation of bone cement distribution and allow timely or early detection of any bone cement leakage in any monitoring direction (Figure 2). Recent research on CT-guided vertebroplasty has shown that, compared with the traditional postoperative radiographic evaluation, vertebroplasty is associated with a higher incidence of smaller and asymptomatic bone cement leakage (Grossterlinden et al., 2009; Amoretti et al., 2010; Doria and Tranquilli Leali, 2010).

With accurate patient selection and careful operation, complications will rarely occur. However, the complication rate in patients with metastatic tumors is apparently higher than that in patients with osteoporosis. Our study showed that CT helped reduce the incidence of mild and severe complications such as spinal cord and nerve root compression caused by epidural leakage of bone cement, pulmonary embolism caused by venous leakage, hematoma formation, rib or vertebral arch pedicle fractures, and pneumothorax. Two patients developed nail path hematoncus, which was possibly associated with old age, and some patients showed impaired coagulation function. In addition, we used a minor incision and did not perform postoperative drainage, which also possibly caused nail path hematoncus. If the operation is performed under strict aseptic conditions and prophylactic antibiotics are administered, infection can be effectively avoided. In the present study, there were no direct injuries caused by the percutaneous puncture and no indirect injuries related to bone cement leakage caused by the resulting vertebral arch pedicle break. However, the leakage will possibly cause more severe disastrous consequence (i.e., palsy). In addition, 22 patients (20.7\%) presented asymptomatic paravertebral bone cement leakage. Although intraoperative and postoperative CT may identify minor bone cement leakage, its incidence was reduced in our study compared with that previously reported. This finding may be related to the working channel that was constructed in the first attempt used in our study, and the bone cement filling at the center of vertebral body. As the puncture is completed at first attempt, sclerotin around the working channel is gradually compressed, reducing the nail path leakage of the bone cement and bone cement filling that deviates from the vertebral body center, increasing the tissue interval and space distance from the surrounding important organs. Moreover, it can reduce bone cement leakage, improve safety of bone cement filling, and provide better mechanical support. For patients with osteoporosis, the vertebral gap leakage is usually caused by the formation of hollows or cracks on the endplates. However, the biomechanical characteristics were unchanged and intervertebral disc degeneration was accelerated.

For patients with metastatic tumors, venous plexus leakage and epidural leakage of the bone cement occur more easily because of tumor angiogenesis (Bennett, 2011). Intravascular bone cement at the anterior vertebral body usually leaks along the rami anastomoticus of the paravertebral venous plexus and eventually enters the vena cava system, which can result in pulmonary embolism. Posterior bone cement leakage occurs outside the dura mater or at the paravertebral venous plexus. Furthermore, bone cement accumulation at this site will cause spinal cord and nerve root compression, thereby resulting in the corresponding symptoms. In summary, bone cement leakage outside the vertebral body generates fewer symptoms. If bone cement leakage is found during intraoperative bone cement filling, the filling is stopped for several seconds to allow bone cement hardening.

In terms of bone cement polymerization, the vertebral body is stable. Clinically, the 
pain in the majority of patients was relieved after a $24-\mathrm{h}$ postoperative period. Previous reports showed that pain relief was experienced by 75 to $82 \%$ of patients with metastatic bone tumors, and 73 to $97 \%$ of patients with osteoporotic compression fractures experienced no pain (Phillips, 2003; Majd et al., 2005; Garfin et al., 2011). The combined pain relief rate (98\%) in metastatic tumor and osteoporosis cases in the current study was similar to the clinical results previously reported.

PKP guided by a combination of CT and C-arm fluoroscopy, with the finger-touch method for determining needle entry point, can determine the ideal operation approach, allow one-time construction of the working channel, increase the operation success rate, reduce the operation time, enhance the accuracy and safety of the PKP operation process, and reduce the incidence of complications.

\section{ACKNOWLEDGMENTS}

Research supported by the Science and Technology Commission Scientific Research Project, Wuhu (\#2011, 47).

\section{REFERENCES}

Amoretti N, Lesbats V, Marcy PY, Hauger O, et al. (2010). Dual guidance (CT and fluoroscopy) vertebroplasty: radiation dose to radiologists. How much and where? Skeletal Radiol. 39: 1229-1235.

Anselmetti GC, Manca A, Hirsch J, Montemurro F, et al. (2011). Percutaneous vertebroplasty in osteoporotic patients: an institutional experience of 1,634 patients with long-term follow-up. J. Vasc. Interv. Radiol. 22: 1714-1720.

Bennett K (2011). Osteoporosis and kyphoplasty. J. Pain Palliat. Care Pharmacother. 25: 286-287.

Chew C, Craig L, Edwards R, Moss J, et al. (2011). Safety and efficacy of percutaneous vertebroplasty in malignancy: a systematic review. Clin. Radiol. 66: 63-72.

Doria C and Tranquilli Leali P (2010). Percutaneous techniques in the treatment of osteoporotic, traumatic and neoplastic fractures of thoraco-lumbar spine: our institutional experience. Injury 41: 1136-1139.

Garfin SR, Yuan HA and Reiley MA (2011). New technologies in spine: kyphoplasty and vertebroplasty for the treatment of painful osteoporotic compression fractures. Spine 26: 1511-1515.

Grossterlinden L, Begemann PG, Lehmann W, Nuechtern J, et al. (2009). Sacroplasty in a cadaveric trial: comparison of CT and fluoroscopic guidance with and without balloon assistance. Eur. Spine J. 18: 1226-1233.

Hide IG and Gangi A (2009). Percutaneous vertebroplasty: history, technique and current perspectives. Clin. Radiol. 59: 461-467.

Kallmes DF and Jensen ME (2003). Percutaneous vertebroplasty. Radiology 229: 27-36.

Katsanos K, Sabharwal T and Adam A (2010). Percutaneous cementoplasty. Semin. Intervent. Radiol. 27: 137-147.

Majd ME, Farley S and Holt RT (2005). Preliminary outcomes and efficacy of the first 360 consecutive kyphoplasties for the treatment of painful osteoporotic vertebral compression fractures. Spine J. 5: 244-255.

Phillips FM (2003). Minimally invasive treatments of osteoporotic vertebral compression fractures: vertebroplasty and kyphoplasty. Spine 28: S45-S53.

Rad AE and Kallmes DF (2011). Correlation between preoperative pain duration and percutaneous vertebroplasty outcome. AJNR Am. J. Neuroradiol. 32: 1842-1845.

Rad AE, Gray LA, Sinaki M and Kallmes DF (2011). Role of physical activity in new onset fractures after percutaneous vertebroplasty. Acta Radiol. 52: 1020-1023.

Shengzhong M, Dongjin W, Shiqing W, Yang S, et al. (2012). Modification of percutaneous vertebroplasty for painful old osteoporotic vertebral compression fracture in the elderly: preliminary report. Injury 43: 486-489.

Smith ZA, Yang I, Gorgulho A, Raphael D, et al. (2012). Emerging techniques in the minimally invasive treatment and management of thoracic spine tumors. J. Neurooncol. 107: 443-455.

Trumm C, Jakobs T, Pahl A, Stahi R, et al. (2012a). CT fluoroscopy-guided percutaneous vertebroplasty in patients with multiple myeloma: analysis of technical results from 44 sessions with 67 vertebrae treated. Diagn. Interv. Radiol. 18: 111-120.

Trumm CG, Pahl A, Helmberger TK, Jakobs TF, et al. (2012b). CT fluoroscopy-guided percutaneous vertebroplasty in 
spinal malignancy: technical results, PMMA leakages, and complications in 202 patients. Skeletal Radiol. 41: 13911400 .

Young C, Munk PL, Heran MK, Lane MD, et al. (2011). Treatment of severe vertebral body compression fractures with percutaneous vertebroplasty. Skeletal Radiol. 40: 1531-1536.

Zampini JM, White AP and McGuire KJ (2010). Comparison of 5766 vertebral compression fractures treated with or without kyphoplasty. Clin. Orthop. Relat. Res. 468: 1773-1780. 